Meta

Journal des traducteurs

Translators' Journal

\title{
Terminologie, sécurité alimentaire et santé publique
}

\section{Bassey E. Antia, Yaya Mahamadou et Tioguem Tamdjo}

Volume 54, numéro 3, septembre 2009

URI : https://id.erudit.org/iderudit/038316ar

DOI : https://doi.org/10.7202/038316ar

Aller au sommaire du numéro

\section{Éditeur(s)}

Les Presses de l'Université de Montréal

ISSN

0026-0452 (imprimé)

1492-1421 (numérique)

Découvrir la revue

Citer cet article

Antia, B. E., Mahamadou, Y. \& Tamdjo, T. (2009). Terminologie, sécurité alimentaire et santé publique. Meta, 54(3), 575-587.

https://doi.org/10.7202/038316ar

\section{Résumé de l'article}

Le présent article interroge la place des analyses terminologiques dans le domaine de l'élevage dans la province de l'Extrême-Nord du Cameroun, qui mérite bien d'être qualifié de stratégique et même de critique en matière de sécurité. En effet, les enjeux sont de taille, puisqu'ils concernent non seulement la sécurité alimentaire et la santé publique, mais aussi d'autres objectifs sociaux. L'article présente une analyse d'un corpus de termes de maladies bovines et de descriptions pertinentes, recueilli dans le cadre de discussions en groupe. Cette analyse, fondée sur un classement des termes et sur l'examen de relations terminologiques (équivalence, synonymie), permet de révéler les structures épistémiques différentes qui sous-tendent et peut-être compliquent la communication entre personnel vétérinaire et éleveurs. Les synonymes qui ne sont pas considérés comme tels par tous les acteurs, et les termes dits équivalents qui ne se recouvrent pas conceptuellement, sont autant d'éléments sous-jacents à une communication dysfonctionnelle en milieu pastoral, avec d'éventuelles conséquences pour la sécurité alimentaire ainsi que pour la santé publique. 


\title{
Terminologie, sécurité alimentaire et santé publique
}

\author{
BASSEY E. ANTIA \\ University of Maiduguri, Maiduguri, Nigeria \\ bantia1@yahoo.co.uk \\ YAYA MAHAMADOU \\ University of Maiduguri, Maiduguri, Nigeria \\ TIOGUEM TAMDJO \\ University of Maiduguri, Maiduguri, Nigeria \\ tamdjo@yahoo.fr
}

\begin{abstract}
RÉSUMÉ
Le présent article interroge la place des analyses terminologiques dans le domaine de l'élevage dans la province de l'Extrême-Nord du Cameroun, qui mérite bien d'être qualifié de stratégique et même de critique en matière de sécurité. En effet, les enjeux sont de taille, puisqu'ils concernent non seulement la sécurité alimentaire et la santé publique, mais aussi d'autres objectifs sociaux. L'article présente une analyse d'un corpus de termes de maladies bovines et de descriptions pertinentes, recueilli dans le cadre de discussions en groupe. Cette analyse, fondée sur un classement des termes et sur l'examen de relations terminologiques (équivalence, synonymie), permet de révéler les structures épistémiques différentes qui sous-tendent et peut-être compliquent la communication entre personnel vétérinaire et éleveurs. Les synonymes qui ne sont pas considérés comme tels par tous les acteurs, et les termes dits équivalents qui ne se recouvrent pas conceptuellement, sont autant d'éléments sous-jacents à une communication dysfonctionnelle en milieu pastoral, avec d'éventuelles conséquences pour la sécurité alimentaire ainsi que pour la santé publique.
\end{abstract}

\begin{abstract}
This article examines the role of terminological analyses in animal husbandry in the Extreme North province of Cameroon, a domain in which the stakes (food security, public health, other social goals) are so high that it can rightly be considered as strategic and safety critical. Based on data from several focus group discussions, the article analyses terms for cattle diseases and descriptions of these diseases from the standpoint of a term sorting task as well as equivalence relations, synonymy. The analysis unlocks differences in the knowledge structures underpinning and probably complicating communication between veterinarians and cattle farmers. Communication comes across as taking place against the backdrop of, among others, contested synonymy and differences in the intension of terms said to be equivalent interlingually. This backdrop underscores the link between dysfunctional communication among actors in animal husbandry and such social goals as food security and public health.
\end{abstract}

\section{MOTS-CLÉS/KEYWORDS}

maladies bovines, modèles explicatifs des maladies, communication entre personnel vétérinaire et éleveurs, communication dysfonctionnelle, Cameroun

cattle diseases, explicative models of disease, communication between veterinarians and farmers, dysfunctional communication, Cameroon 


\section{Portrait du monde pastoral}

Depuis un certain temps, la santé des animaux fait la une du discours public. Les ravages causés par l'épizootie d'encéphalopathie spongiforme bovine (la maladie de la vache folle) et, plus récemment, par celle de la fièvre aphteuse, nous ont montré à quel point nous sommes vulnérables tant sur le plan de la santé publique que sur celui de la sécurité alimentaire. Dans tous les pays touchés par la crise de la fièvre aphteuse survenue en 2001, les coûts encourus ont été énormes: la valeur des centaines de milliers de vaches, de moutons et de porcs abattus et brûlés; le coût même des abattages; le coût des embargos; enfin, les coûts psychologiques impossibles à évaluer en termes monétaires. Au Royaume-Uni, la crise de la fièvre aphteuse de 2001 aurait fait perdre 7 milliards de dollars à l'industrie du tourisme et 5 milliards de dollars à l'industrie agricole (Sécurité publique Canada 2006).

Dans différents pays en voie de développement, sinon ailleurs, l'élevage comporte beaucoup de caractéristiques communes, lesquelles, selon Fraser et Restrepo-Estrada (1998), se retrouveraient aussi dans les secteurs analogues tels que l'agriculture, la pêche, etc. Il s'agit, entre autres, des éléments suivants:

a) productivité en baisse à cause des maladies, pertes importantes en vies animales, etc.;

b) conflit épistémique entre pratiques traditionnelles et techniques modernes/occidentales: on se voit expliquer, par des agents de développement, que les pratiques d'antan sont à écarter et doivent céder la place à d'autres, plus rentables et plus respectueuses de l'environnement;

c) agents ou conseillers peu motivés parce que mal payés, préférant habiter la ville plutôt que la campagne où ils sont en poste, se compromettant sans scrupules sur le plan de l'éthique humaine aussi bien que professionnelle, tant que se profilent des avantages pécuniaires;

d) emplois différents d'une même langue ou usage de langues différentes par les agents d'intervention. Soit ils ne parlent pas la langue de ceux auxquels ils s'adressent, soit ils imprègnent cette langue de concepts et de termes appris en formation et inconnus des interlocuteurs;

e) moyens financiers et matériaux incompatibles avec les besoins et infrastructures inadéquates (Fraser et Restrepo-Estrada 1998).

$\mathrm{Si}$, pour des raisons liées à ces facteurs ou à d'autres (force majeure, ignorance, incompétence), une épizootie quelconque survenait, les acteurs impliqués dans l'élevage de plusieurs pays en voie de développement seraient probablement pris au dépourvu, voire incapables de prendre les mesures appropriées pour la contenir. Et il a été bel et bien question d'incompétence lors de l'épizootie de fièvre aphteuse de 2001, à en croire des avis comme celui-ci: "L'épizootie de fièvre aphteuse, qui a décimé les élevages britanniques (...) a été le résultat d'une série de retards, de défaillances, d'incompétences et de dysfonctionnements» (cité dans le Bulletin des agriculteurs 2002). Étant donné l'instinct de survie des éleveurs dans les pays en voie de développement, éleveurs qui reçoivent rarement des subventions de l'État en contexte d'épizootie, il y a de fortes chances que les faibles structures de surveillance en place favorisent des activités dangereuses pour la santé publique: refus d'abattre les animaux atteints de la maladie, vente clandestine des animaux ou de produits (viande et lait) contaminés.

L'un des principes de la «communication pour le développement» (Frasier et Restrepo-Estrada 1998: 5) consiste à faire prendre conscience aux agents de trans- 
formation (décideurs, réalisateurs, individus en société) les conséquences de leur réalité actuelle, et cela, par le biais de stratégies nouvelles de sensibilisation. L'objectif du présent article est de montrer comment un ensemble d'analyses terminologiques pourrait aider à sensibiliser tous les acteurs de l'univers pastoral à leur réalité, tant à l'échelle nationale qu'internationale, et ainsi, à stimuler des initiatives qui pourront (mieux) assurer la sécurité alimentaire et la santé publique dans un monde où les catastrophes ne connaissent pas de frontières.

\section{Que peut la terminologie?}

La communication en contexte d'élevage (y compris celui, plus restreint, des maladies bovines) est un exemple de discours ou de langue de spécialité. Malheureusement, le mot langue empêche souvent de voir que l'intervention du linguiste dans un domaine de connaissances spécialisées ne se réduit ni à des questions de correction grammaticale, ni à l'inventaire des parties du discours, comme ce fut le cas dans les premières recherches linguistiques sur le discours spécialisé.

L'intervention du linguiste-terminologue s'étend aux connaissances spécialisées elles-mêmes. Il y a bien longtemps qu'Albert Einstein et George Orwell disaient, respectivement, que la substance même de nos connaissances sur un sujet donné réside dans la terminologie pertinente, et que celui qui maîtrise la terminologie maîtrise la matière (cité dans Antia 2000: 100). Pour se convaincre du statut de pierre angulaire assumé par les termes en discours spécialisé, il suffit de mener deux expériences portant sur un texte technique. Dans la première, on cache ou on efface tous les mots à contenu spécialisé, pour, ensuite, demander à un expert du domaine en question de combler les vides. Dans la deuxième expérience, ce sont seulement les mots à contenu spécialisé qui restent dans le texte, tous les autres mots (y compris les mots grammaticaux) ayant été effacés ou cachés. Souvent, pour ne pas dire dans $100 \%$ des cas, c'est cette configuration de texte qui produit des réponses intelligentes; en d'autres termes, contrairement à la première configuration de texte, la deuxième permet de mieux deviner le contenu du texte initial.

Ce n'est donc pas fortuitement que les termes vont nous servir à déverrouiller les structures épistémiques de l'univers pastoral, un univers à deux planètes dont l'une est habitée par des éleveurs et l'autre par le personnel vétérinaire, médecins et infirmiers. C'est aux médecins et aux infirmiers vétérinaires qu'est confiée la tâche de «communiquer» avec les éleveurs pour que soient réalisés différents objectifs sociaux dont: le maintien de troupeaux sains; la production adéquate de viande, du lait et d'autres dérivés; le maintien de conditions sanitaires adéquates; la création d'emplois; etc. Cependant, il y a aussi des objectifs individuels qui ne sont pas seulement de nature économique. Souvent, l'activité pastorale obéit à une éthique traditionnelle qui se justifie par le rayonnement que représente la possession d'un cheptel important.

\section{Questions de recherche}

Afin de savoir jusqu'à quel point les structures épistémiques des éleveurs et des vétérinaires/infirmiers vétérinaires se recoupent (en matière de maladies), nous avons posé trois questions: 
a) Comment se présente le rapport concept-terme de part et d'autre, notamment lorsque les deux planètes, éleveurs d'un côté et médecins/infirmiers vétérinaires de l'autre, se confrontent?

b) Les maladies dites équivalentes dans chacune des planètes se recouvrent-elles conceptuellement?

c) Les éleveurs et le personnel vétérinaire accordent-ils la même priorité à certaines maladies?

Répondre à ces questions devrait nous permettre d'émettre des hypothèses sur la nature de la communication en milieu pastoral, d'en tirer des conséquences et, ce faisant, de sensibiliser décideurs, réalisateurs et les communautés d'éleveurs.

\section{Méthodologie}

Les données pour cette étude ont été recueillies en avril 2001 dans la province de l'Extrême-Nord du Cameroun. Cette région limitrophe du Nigeria est celle où l'élevage est le plus pratiqué au Cameroun. Ce sont les Foulbés (connus aussi sous le nom de Peuls) qui le pratiquent. Ils parlent le foulfouldé. Un premier ensemble de données a été collecté à Djiddel, village des environs de Bago. Douze éleveurs (âgés de 20 à 66 ans et possédant de 4 à 46 bœufs) ont été réunis dans la cour du chef de village en présence de six notables pour deux séances de discussion en groupe. Dans une première séance, il s'agissait d'obtenir une liste des maladies bovines les plus répandues dans la région, ainsi qu'une description exhaustive des symptômes de ces maladies et des thérapies pratiquées. Le cas échéant, les synonymes pour les noms des maladies devaient aussi être précisés. La deuxième séance de discussion en groupe avec les mêmes personnes était consacrée à leur perception de la gravité des maladies mentionnées lors de la première séance. Les maladies devaient être rangées par ordre décroissant de gravité, cette dernière pouvant être définie en termes qualitatifs ou quantitatifs.

Des séances analogues ont été organisées à Maroua, la capitale provinciale, avec des médecins vétérinaires (au nombre de trois), puis avec des infirmiers vétérinaires (au nombre de quatre). Les discussions avec ces spécialistes se sont déroulées en français, bien que les participants soient des Foulbés. L'un des chercheurs (YM) prenait des notes, ce qui permettait de vérifier, avant la fin de la séance, le contenu des discussions.

Comme dans tout travail de terminologie comparée (Arntz et Picht 1995), il fallait obtenir les données séparément (même quand il s'agissait des médecins vétérinaires et des infirmiers) et dans la langue usuelle de travail des participants. Toutefois, les infirmiers se sont vu demander comment ils appelleraient en français les maladies mentionnées en foulfouldé par les éleveurs, cela parce que ce sont eux qui vont dans les campagnes sensibiliser ces derniers ou vacciner les bovins. Puisque les éleveurs ne parlent que le foulfouldé, les infirmiers (formés en français) doivent en principe essayer de se faire comprendre. L'intension et le sens des termes proposés en français correspondraient-ils à la description conceptuelle des maladies en cause faite et par les éleveurs et (dans une tâche précédente) par les infirmiers?

Pour résumer, nous avions trois catégories de données: description des maladies identifiées, perception de la gravité des maladies et enfin, équivalents français des noms foulfouldés de maladies. 


\section{Résultats et discussion}

Les données de base utilisées pour l'analyse sont présentées dans les tableaux 1 et 2. Le tableau 1 présente la description des treize maladies identifiées par les éleveurs, c'est-à-dire les modèles d'explication locaux associés à ces maladies.

TABLEAU 1

\section{Noms et descriptions des maladies selon les éleveurs}

\begin{tabular}{|c|c|c|}
\hline Nom de la maladie & Symptômes & Cause, traitement, prévention, etc. \\
\hline $\begin{array}{l}\text { 1. Bodewou ou } \\
\text { Petou }\end{array}$ & $\begin{array}{l}\text { a) Diarrhées sanguinolentes. } \\
\text { b) Yeux très rouges. }\end{array}$ & $\begin{array}{l}\text { a) Découper } 2 \text { noix de cola (surtout blanches) } \\
\text { en sept morceaux, lire les sourates } 112 \text { et } 1 \text { du } \\
\text { Coran sur les pièces découpées. Les faire } \\
\text { bouillir dans une marmite dans laquelle la } \\
\text { cuisson du repas du soir vient de se terminer. } \\
\text { Faire boire la bête malade et le reste du } \\
\text { troupeau le lendemain matin. } \\
\text { b) Écraser un épi de mil vieux de deux } \\
\text { saisons et le faire avaler à l'animal et au reste } \\
\text { du bétail. }\end{array}$ \\
\hline 2. Kikoyel & $\begin{array}{l}\text { a) La bête est faible. } \\
\text { b) Elle traîne toujours derrière le } \\
\text { troupeau. } \\
\text { c) Dès qu'on touche l'animal, il } \\
\text { sursaute et tombe en battant des } \\
\text { pattes, avant de mourir brutalement. } \\
\text { d) La rate est enflée (à l'autopsie). }\end{array}$ & $\begin{array}{l}\text { a) Porter un fer au rouge par le feu et brûler } \\
\text { toutes les articulations. } \\
\text { b) Faire bouillir les écorces d'un caïlcedra et } \\
\text { faire boire l'animal. } \\
\text { c) La maladie est très contagieuse. }\end{array}$ \\
\hline 3. Mbalki & $\begin{array}{l}\text { a) L'animal manque d'appétit. } \\
\text { b) Il n'arrive pas à marcher. } \\
\text { c) Il refuse l'eau jusqu'à en mourir. }\end{array}$ & La maladie est incurable. \\
\hline 4. $\underline{\text { Damal }}$ & $\begin{array}{l}\text { a) L'animal commence par manquer } \\
\text { d'appétit. } \\
\text { b) Il refuse complètement de } \\
\text { s'alimenter parfois jusqu'à } 3 \text { à } 4 \text { jours, } \\
\text { souvent même plus, puis il meurt. } \\
\text { c) La rate est "pourrie» (c'est-à-dire } \\
\text { nécrosée; à l'autopsie). }\end{array}$ & $\begin{array}{l}\text { a) La maladie est traditionnellement } \\
\text { incurable. } \\
\text { b) Il faut voir les autorités sanitaires sur } \\
\text { place. } \\
\text { c) La maladie est très dangereuse. }\end{array}$ \\
\hline 5. Mborou/Djobou & $\begin{array}{l}\text { a) Écoulement de salive en grande } \\
\text { quantité par la gueule et les narines. } \\
\text { b) Blessures au niveau des onglons. } \\
\text { c) L'animal piaffe constamment en } \\
\text { dégageant une forte odeur. } \\
\text { d) Très souvent, des blessures graves } \\
\text { font perdre la langue. } \\
\text { N. B. Les symptômes de Djobou } \\
\text { comportent en plus des «aphtes» } \\
\text { dans le cou. }\end{array}$ & $\begin{array}{l}\text { a) Appliquer du miel sur la surface des } \\
\text { parties infectées. } \\
\text { b) Selon cinq éleveurs, cette maladie est } \\
\text { incurable par la médecine moderne. } \\
\text { c) Malgré sa gravité, la maladie n'empêche } \\
\text { pas la fertilité des femelles. }\end{array}$ \\
\hline 6. Boutou & $\begin{array}{l}\text { a) Gonflement des muscles. } \\
\text { b) L'animal ne peut même pas aller } \\
\text { pâturer. }\end{array}$ & $\begin{array}{l}\text { Brûler toutes les articulations avec un fer } \\
\text { rougi au feu. }\end{array}$ \\
\hline 7. Fouloukké & $\begin{array}{l}\text { a) Gonflement du ventre. } \\
\text { b) Manque d'appétit. } \\
\text { c) Malgré tout, la bête continue de } \\
\text { ruminer imperturbablement. }\end{array}$ & $\begin{array}{l}\text { a) Piler des feuilles sèches ou fraîches de } \\
\text { baobab, ajouter du piment, mettre dans l'eau } \\
\text { et faire boire l'animal. } \\
\text { b) Brûler le ventre gonflé avec un fer rougi au } \\
\text { feu. }\end{array}$ \\
\hline 8. Boumsoudé & $\begin{array}{l}\text { a) Gonflement du ventre. } \\
\text { b) Respiration difficile. } \\
\text { c) Les poumons sont complètement } \\
\text { "pourris» (nécrosés à l'autopsie). }\end{array}$ & $\begin{array}{l}\text { a) Lorsqu'une bête meurt à cause de cette } \\
\text { maladie, on découpe ses poumons en petits } \\
\text { morceaux et on les place juste sous l'oreille } \\
\text { gauche de toutes les bêtes encore saines en } \\
\text { les blessant un tout petit peu. } \\
\text { b) Maladie très dangereuse. }\end{array}$ \\
\hline
\end{tabular}




\begin{tabular}{|c|c|c|}
\hline 9. Douslaré & $\begin{array}{l}\text { a) Elle n'attaque que les veaux. } \\
\text { b) C'est une maladie qui ressemble au } \\
\text { paludisme. } \\
\text { c) Le veau meurt subitement quand il } \\
\text { boit de l'eau pendant les deux } \\
\text { premiers jours de la maladie. } \\
\text { d) Une bête est atteinte une seule fois } \\
\text { durant toute sa vie. }\end{array}$ & $\begin{array}{l}\text { a) Le veau peut être gardé au soleil. } \\
\text { b) Brûler toutes les articulations à l'aide d'un } \\
\text { fer rougi au feu. } \\
\text { c) Quand on constate que l'animal a très soif, } \\
\text { on lui fait boire de l'eau chaude. }\end{array}$ \\
\hline 10. Miri & $\begin{array}{l}\text { a) Présence de punaises sur le corps } \\
\text { de l'animal. } \\
\text { b) Il peut perdre tous ses poils } \\
\text { jusqu'aux pattes. }\end{array}$ & $\begin{array}{l}\text { Piler des feuilles de la plante Worba } \\
\text { (semblable à l'oseille) et appliquer sur tout le } \\
\text { corps de l'animal. }\end{array}$ \\
\hline 11. Baccalé & $\begin{array}{l}\text { a) L'animal boite en marchant. } \\
\text { b) Des gonflements sont observés } \\
\text { partout sur le corps. } \\
\text { c) La bête souffre, mais cela } \\
\text { n'empêche pas la reproduction. }\end{array}$ & $\begin{array}{l}\text { Faire bouillir un arbuste appelé Mbalchi et } \\
\text { en donner à boire à la bête. }\end{array}$ \\
\hline $\begin{array}{l}\text { 12. Boudi ou Dousé } \\
\text { Boulé }\end{array}$ & $\begin{array}{l}\text { a) L'animal mange bien, mais il } \\
\text { maigrit de jour en jour. } \\
\text { b) Les yeux coulent. } \\
\text { c) Des pustules contenant du pus sont } \\
\text { observées un peu partout sur le corps } \\
\text { de la bête. }\end{array}$ & $\begin{array}{l}\text { a) Faire bouillir de l'écorce de caïlcedra et } \\
\text { faire boire l'animal (un litre environ). } \\
\text { b) Faire sécher de l'écorce du même arbre et } \\
\text { les mélanger avec un aliment que l'animal } \\
\text { pourra facilement consommer. }\end{array}$ \\
\hline 13. Gaouyel & $\begin{array}{l}\text { a) Une maladie extraordinaire et } \\
\text { dangereuse, parce que l'animal peut } \\
\text { sortir bien portant le matin, puis } \\
\text { mourir pendant le pâturage. } \\
\text { b) L'animal présente souvent des } \\
\text { gonflements sur le corps, de couleur } \\
\text { noire, qui font du bruit au toucher. }\end{array}$ & $\begin{array}{l}\text { a) À une certaine époque, les éleveurs } \\
\text { accusaient les mauvais esprits et quittaient } \\
\text { les lieux. } \\
\text { b) Soumettre tout le troupeau à une } \\
\text { fumigation avec des feuilles fraîches de } \\
\text { tamarinier et répéter pendant plusieurs } \\
\text { jours. } \\
\text { c) Généralement, c'est une maladie causée } \\
\text { par l'arrêt brusque des pluies. }\end{array}$ \\
\hline
\end{tabular}

Le tableau 1 met en évidence le caractère souvent magico-religieux du modèle utilisé par les éleveurs pour les maladies 1, 6, 7, 8 et 13. Les mauvais esprits sont impliqués dans l'étiologie de quelques-unes; la gamme des remèdes va de l'administration de noix de cola, associée à des lectures coraniques, à des mutilations de l'animal (découpage des poumons, brûlure des parties affectées avec un fer rougi au feu). Il y a lieu d'étudier ces pratiques de près afin de déterminer leur efficacité. En attendant, on pourra observer le peu de confiance dans la médecine occidentale/moderne que semblent avoir certains éleveurs. Parmi les treize maladies décrites, c'est par rapport à une seule (Damal) qu'il est question de recourir aux autorités sanitaires pour des thérapies. Le défi que représente une intervention par les institutions sanitaires publiques est clair: comment ou jusqu'à quel point est-ce que le modèle d'explication magico-religieuse constitue un obstacle aux actions envisagées?

Par ailleurs, alors que les éleveurs identifient treize maladies principales dans la région, les vétérinaires et les infirmiers en identifient seulement neuf. Leurs descriptions par les vétérinaires et les infirmiers étant identiques, elles sont toutes réunies dans le tableau 2. 
TABLEAU 2

Noms et descriptions des maladies selon les vétérinaires ou les infirmiers vétérinaires

\begin{tabular}{|c|c|c|}
\hline Nom de la maladie & Symptômes & Cause, traitement, prévention, etc. \\
\hline 1. Pasteurellose & $\begin{array}{l}\text { a) Maigreur. } \\
\text { b) Diarrhée constante. } \\
\text { c) Présence d'œè̀me dans la gorge. } \\
\text { d) Respiration sifflante. } \\
\text { e) L'animal peut paraître en bonne santé en } \\
\text { sortant du campement, puis mourir le soir. }\end{array}$ & $\begin{array}{l}\text { Tétracyclines. } \\
\text { Prévention: vaccin Pastovax }{ }^{\circledast} .\end{array}$ \\
\hline $\begin{array}{l}\text { 2. Charbon } \\
\text { symptomatique }\end{array}$ & $\begin{array}{l}\text { a) Présence d'œdèmes crépitants au niveau } \\
\text { des masses musculaires (épaule ou cuisse). } \\
\text { b) Quand on ouvre ces œèmeses, on trouve } \\
\text { une espèce de mousse entourant une tache } \\
\text { noirâtre. }\end{array}$ & $\begin{array}{l}\text { Antibiotiques. } \\
\text { Prévention: vaccin Symptovax }{ }^{\oplus} \text {. }\end{array}$ \\
\hline 3. Charbon bactéridien & $\begin{array}{l}\text { a) Présence d'œdèmes, non crépitants. } \\
\text { b) Quand on les ouvre, tout est noir. }\end{array}$ & Antibiotiques. \\
\hline 4. Péripneumonie & $\begin{array}{l}\text { a) Les poumons sont attaqués. } \\
\text { b) La plèvre s'épaissit. } \\
\text { c) Un poumon est plus atteint que l'autre. } \\
\text { d) Respiration difficile. } \\
\text { e) Toux grasse. } \\
\text { f) Écoulement nasal. } \\
\text { g) Asthénie. }\end{array}$ & $\begin{array}{l}\text { Antibiotiques. } \\
\text { Prévention: vaccin Perivax }{ }^{\oplus} \text {. }\end{array}$ \\
\hline 5. Tuberculose & $\begin{array}{l}\text { a) Maigreur. } \\
\text { b) Toux. } \\
\text { c) Gonflement des ganglions. } \\
\text { d) Diarrhée. }\end{array}$ & $\begin{array}{l}\text { Défense de traiter. Abattre l'animal } \\
\text { malade le plus vite possible. }\end{array}$ \\
\hline 6. Trypanosomiase & $\begin{array}{l}\text { a) Fatigue constante. } \\
\text { b) Larmoiement. } \\
\text { d) Chute des poils de la queue. } \\
\text { d) Maigreur et anémie. } \\
\text { e) Manque d'appétit. }\end{array}$ & $\begin{array}{l}\text { Les trypanocides, tels que les } \\
\text { marques suivantes: } \\
\text { - Veriben } \\
\text { - Trypan } \\
\text { - Novidium } \\
\text { - Berenil } \\
{ }^{\infty}\end{array}$ \\
\hline 7. Douve & $\begin{array}{l}\text { a) Diarrhée très nauséabonde. } \\
\text { b) Maigreur. } \\
\text { c) Fatigue constante. }\end{array}$ & $\begin{array}{l}\text { Les douvicides tels que: } \\
\text { - Dovenix }{ }^{\otimes} \text {. }\end{array}$ \\
\hline 8. Fièvre aphteuse & $\begin{array}{l}\text { a) La maladie attaque tous les bovins, mais } \\
\text { ce sont les veaux qui en meurent le plus. } \\
\text { b) Salivation abondante. } \\
\text { c) Pustules dans la bouche. } \\
\text { d) Plaies autour des onglons et dans l'espace } \\
\text { interdigital. } \\
\text { e) Aphtes sur les mamelles. }\end{array}$ & $\begin{array}{l}\text { C'est une maladie bénigne qui se } \\
\text { guérit seule*. }\end{array}$ \\
\hline 9. Brucellose & a) Gonflement des articulations (hygroma). & Antibiotiques. \\
\hline
\end{tabular}

*C'est une erreur qui est difficile à expliquer. La fièvre aphteuse est connue pour ses ravages, donc elle ne peut en aucun cas être considérée comme bénigne. Pour des erreurs analogues, toujours dans le contexte vétérinaire, voir Antia et Kwasari (2009, sous presse).

On peut d'emblée remarquer que la différence dans le nombre de maladies mentionnées par les éleveurs et les vétérinaires/infirmiers suggère des différences épistémiques.

Le tableau 3 présente un classement des maladies bovines réalisé en fonction de la perception de leur gravité d'un côté par les éleveurs, de l'autre par les vétérinaires et les infirmiers vétérinaires. En ce qui concerne la gravité (définie en termes qualitatifs ou quantitatifs), seules cinq maladies (dites «maladies de la première classe» par les éleveurs) soulèvent des inquiétudes de part et d'autre. Les autres maladies, de l'aveu des deux groupes, revêtent moins d'importance. Les vétérinaires et les infirmiers 
vétérinaires ne se sont pas donné la peine de les mentionner (voir le tableau 2 pour la liste intégrale des maladies mentionnées).

TABLEAU 3

Classement des maladies bovines en fonction de la gravité perçue et équivalents foulfouldés français

\begin{tabular}{|c|c|}
\hline Éleveurs & Vétérinaires - infirmiers vétérinaires \\
\hline 1. Bodewu ou petou (peste bovine) & 1. Péripneumonie (Boumsoudé) \\
\hline 2. Boumsoudé (péripneumonie) & 2. Peste bovine ( $\underline{\text { Bodewu }})$ - éradiqué \\
\hline 3. Damal (charbon bactéridien) & 3. Charbon bactéridien ( $\underline{\text { Damal) }}$ \\
\hline 4. Gaouyel (charbon symptomatique) & 4. Charbon symptomatique (Gaouyel/Boutou) \\
\hline 5. Kikoyel (pasteurellose) & 5. Pasteurellose (Kikoyel) \\
\hline \multicolumn{2}{|l|}{ 6. Boudi ou Dousé Boulé (genre d'inflammation) } \\
\hline \multicolumn{2}{|l|}{ 7. Boutou (charbon symptomatique) } \\
\hline \multicolumn{2}{|l|}{ 8. Miri (tiques/gales) } \\
\hline \multicolumn{2}{|l|}{ 9. Baccalé (brucellose) } \\
\hline \multicolumn{2}{|l|}{ 10. Fouloukké (fièvre) } \\
\hline \multicolumn{2}{|l|}{ 11. Mborou ou Djobou (fièvre aphteuse) } \\
\hline \multicolumn{2}{|l|}{ 12. Mbalki (douve) } \\
\hline 13. Douslaré (constipation et trypanosomiase) & \\
\hline
\end{tabular}

Passons à présent à nos trois questions. La première porte sur le rapport conceptterme. Deux situations se dégagent du tableau 3 et de la comparaison des tableaux 1 et 2 :

a) un terme français correspondant à deux termes foulfouldés (par exemple, Gaouyel et Boutou pour le charbon symptomatique, Mborou et Djobou pour la fièvre aphteuse, Bodewou et Petou pour la peste bovine);

b) un terme foulfouldé dénote deux concepts distincts en français (par exemple, Douslaré pour la trypanosomiase et la constipation).

Dans la première situation, il faut remarquer que Mborou et Djobou, d'une part, et Bodewou et Petou, d'autre part, sont des couples de synonymes proposés par les éleveurs eux-mêmes, et qui correspondent chacun à une seule description (voir aussi le tableau 1). Au contraire, Gaouyel et Boutou, pour les éleveurs, correspondent à deux maladies différentes, alors que pour les infirmiers, il s'agit d'une seule et même maladie.

Dans le cadre d'une intervention, un problème peut se poser si les responsables emploient seulement l'un de ces deux termes en s'adressant à des éleveurs qui en rétrécissent l'étendue, parce qu'ils en connaissent un autre qu'ils associent à un autre concept. Ainsi, l'éleveur à qui on défend d'introduire dans la chaîne alimentaire les bœufs atteints de «charbon symptomatique» (Gaouyel) ne soumettra évidemment pas la partie de son troupeau atteinte de Boutou au même régime sanitaire. Une situation analogue, décrite par Antia et Yassin (2001), souligne les conséquences néfastes possibles quand les nosologies (classement des maladies) sont différentes et se traduisent par un rapport concept-terme non univoque, un rapport qui est inconnu des deux parties en présence ou d'une seule (surtout les éleveurs). Au Honduras, dans les campagnes contre la diarrhée, on conseillait à des mères de donner à leur bébé, chaque fois que celui-ci avait la diarrhée, du Litrosol, une solution de réhydratation. 
Le terme employé dans la langue locale pour //diarrhée// était perçu par certaines mères comme se référant à une sorte de diarrhée seulement, puisque dans leur nosologie traditionnelle, elles en identifiaient deux. La solution de réhydratation n'était donc pas donnée aux bébés souffrant du type de diarrhée non explicité, qui en mourraient. Ce n'est que plus tard que les textes des campagnes d'information ont été modifiés pour tenir compte de la nosologie traditionnelle.

Dans la deuxième question, il s'agissait de voir si les concepts correspondant, respectivement, aux dénominations des maladies en foulfouldé et aux dénominations françaises dites équivalentes se recouvrent. On se rappellera que les infirmiers vétérinaires ont établi des équivalences entre les dénominations dans les deux langues en présence (tableau 3). Formés en français, les infirmiers sont les ponts entre les institutions sanitaires publiques et les éleveurs ne parlant que le foulfouldé. Nous avons déjà souligné que les éleveurs identifient treize maladies réputées très répandues alors que les vétérinaires en proposent neuf (tableaux 1 et 2). Certaines maladies mentionnées par un groupe ne figurent pas sur la liste de l'autre, et vice versa. Le tronc commun est constitué de neuf maladies. Nous aurons donc affaire à neuf paires de dénominations.

Le tableau 4 présente une synthèse des données pertinentes à ce tronc commun, dégagées des tableaux 1, 2 et 3. Ce qui nous intéresse, c'est le nombre de traits conceptuels (symptômes) partagés par chacun des concepts dénotés par les différentes paires de dénominations. L'évaluation de la similarité des concepts est évaluée grâce au calcul suivant: pour chaque paire, le nombre de symptômes le plus important fournit le dénominateur, tandis que le numérateur est obtenu en comptant le nombre de symptômes partagés. Les symptômes sont présentés, de part et d'autre, comme ils ont été proposés, car l'ordre pourrait renseigner sur les symptômes considérés comme les plus saillants, voire pathognomoniques. Finalement, on remarquera qu'une certaine souplesse sous-tend les liens que nous établissons entre les symptômes. Par exemple, nous considérons une cause (le manque d'appétit), qui est le trait conceptuel d'un terme foulfouldé, comme équivalant à un effet (la faiblesse), qui est associé au terme français; ou encore, nous procédons à un déplacement spatial, en mettant en relation le gonflement du ventre et l'épaississement de la plèvre.

TABLEAU 4

Correspondance conceptuelle des termes foulfouldés et des termes français dits équivalents: symptômes partagés

\begin{tabular}{|c|c|}
\hline Descriptions des éleveurs & Descriptions des vétérinaires/infirmiers \\
\hline$\frac{\text { Kikoyel }}{\text { a) La bête est faible. }}$ & $\begin{array}{l}\text { Pasteurellose } \\
\text { a) Maigreur. }\end{array}$ \\
\hline \multicolumn{2}{|c|}{$\mathrm{a}=\mathrm{a} ; 1$ symptôme partagé sur 5 , soit $20 \%$} \\
\hline $\begin{array}{l}\text { Mbalki } \\
\text { a) L'animal manque d'appétit. } \\
\text { b) Il n'arrive pas à marcher. }\end{array}$ & $\begin{array}{l}\text { Douve } \\
\text { b) Maigreur. } \\
\text { c) Fatigue constante. }\end{array}$ \\
\hline \multicolumn{2}{|c|}{$a=b ; b=c ; 2$ symptômes partagés sur 3 , soit $66 \%$} \\
\hline Damal & Charbon bactéridien \\
\hline \multicolumn{2}{|c|}{ Aucun symptôme n'est partagé, soit $0 \%$} \\
\hline
\end{tabular}




\section{Mborou/Djobou}

a) Écoulement de salive en grandes quantités par la gueule et les narines.

b) Blessures au niveau des onglons.

e) N. B.: Les symptômes de Djobou sont identiques, avec en plus des «aphtes» dans le cou.
Fièvre aphteuse

b) Salivation abondante.

d) Plaies autour des onglons et dans l'espace interdigital.

e) «Aphtes» sur les mamelles.

\section{$\mathrm{a}=\mathrm{b} ; \mathrm{b}=\mathrm{d} ; \mathrm{e}=\mathrm{e} ; 3$ symptômes partagés sur 5 , soit $60 \%$}

\begin{tabular}{|l|l}
\hline $\begin{array}{l}\text { Boutou } \\
\text { a) Gonflement des muscles. }\end{array}$ & $\begin{array}{l}\text { Charbon symptomatique } \\
\text { a) Présence d'œdèmes crépitants au niveau des } \\
\text { masses musculaires (épaule ou cuisse). }\end{array}$ \\
\hline
\end{tabular}

$\mathrm{a}=\mathrm{a} ; 1$ symptôme partagé sur 5 , soit $20 \%$

\section{Boumsoudé}

a) Gonflement du ventre.

b) Respiration difficile.

c) Les poumons sont complètement "pourris» (à l'autopsie).
Péripneumonie

b) La plèvre devient épaisse.

d) La respiration est difficile.

a) Les poumons sont attaqués. b) La plèvre devient épaisse.

\begin{tabular}{|c|c|}
\hline \multicolumn{2}{|c|}{$\mathrm{a}=\mathrm{b} ; \mathrm{b}=\mathrm{d} ; \mathrm{c}=\mathrm{a} / \mathrm{b} ; 3$ symptômes partagés sur 7 , soit $42 \%$} \\
\hline Douslaré & Trypanosomiase + constipation \\
\hline \multicolumn{2}{|c|}{ Aucun symptôme n'est partagé, soit $0 \%$} \\
\hline $\begin{array}{l}\text { Baccalé } \\
\text { a) L'animal boîte en marchant. } \\
\text { b) Gonflements partout sur le corps. }\end{array}$ & $\begin{array}{l}\text { Brucellose } \\
\text { a) Gonflement des articulations (hygroma). }\end{array}$ \\
\hline \multicolumn{2}{|c|}{$\mathrm{a} / \mathrm{b}=\mathrm{a} ; 2$ symptômes partagés sur 3 , soit $66 \%$} \\
\hline $\begin{array}{l}\text { Gaouyel } \\
\text { a) Une maladie extraordinaire et dangereuse } \\
\text { parce que l'animal peut sortir le matin bien } \\
\text { portant et mourir pendant le pâturage. }\end{array}$ & $\begin{array}{l}\text { Charbon symptomatique (une } 2^{e} \text { fois) } \\
\text { a) Présence d'œdèmes crépitants au niveau des } \\
\text { masses musculaires (épaule ou cuisse). } \\
\text { b) Quand on ouvre ces œè̀mes, il y a une } \\
\text { espèce de mousse qui entoure une tache } \\
\text { noirâtre. }\end{array}$ \\
\hline
\end{tabular}

Ces données montrent que sur neuf paires de dénominations, les concepts dénotés par cinq d'entre elles se recouvrent à moins de $50 \%$. Parmi ces cinq paires, on en compte deux où la coïncidence des traits conceptuels est nulle. Apportons quelques précisions. Si l'on n'écarte pas la possibilité qu'il existe d'autres symptômes qui auraient rehaussé le degré d'équivalence, on doit émettre l'hypothèse que les symptômes mentionnés sont probablement les plus saillants et constituent, de ce fait, des signes prototypiques au service d'une identification rapide. Signalons aussi que la convergence serait encore moindre si l'on tenait compte des traitements éventuels. Les maladies appelées Damal, Mbalki et Mborou sont réputées incurables (y compris par des moyens magico-religieux) alors que les affections présumées y correspondre (charbon bactéridien, douve et fièvre aphteuse, respectivement) se soignent. Finalement, notons non seulement la coïncidence de traits conceptuels, mais aussi l'opposition de certains d'entre eux: ainsi, la maladie nommée Baccalé n'empêcherait pas la reproduction, alors que la maladie, dite équivalente, nommée brucellose provoquerait des avortements.

Certes, ces différences peuvent s'expliquer de nombreuses façons - par exemple, des moyens descriptifs inégaux liés eux aussi à une évolution différente des connaissances, des priorités différentes, etc. Mais il n'est pas difficile d'imaginer les incidences 
de ces différences sur la communication. Comment, avec de tels éléments, peut-on communiquer efficacement de façon à assurer la santé des troupeaux, une production adéquate de viande, de lait et d'autres produits dérivés, ainsi que la santé du public consommateur? Souvent pressés de regagner la ville avant le crépuscule, les infirmiers négligent parfois d'examiner physiquement les animaux, ce qui permettrait de repérer d'éventuelles confusions terminologiques et d'aboutir à un diagnostic correct.

Si l'éleveur affirme que son animal est atteint du Kikoyel, maladie que l'infirmier vétérinaire considère comme la pasteurellose, l'animal sera probablement soigné avec les médicaments spécifiques de cette dernière, à savoir la sulphadimidine ou des antibiotiques à large spectre. Si le diagnostic s'avérait incorrect, c'est-à-dire que l'animal ne souffre pas de pasteurellose, mais qu'il est infecté par la douve (qui ressemble à la précédente par certains aspects, mais qui se soigne avec le nitroxynil ou le rafoxonide), l'emploi de traitements inadéquats pourrait entraîner différents problèmes.

Il faut tout d'abord évoquer la question de la résistance aux médicaments. Comme le traitement administré (sulphadimidine ou antibiotiques à large spectre) est dirigé contre la pasteurellose, qui n'est en fait pas la maladie en cause, l'agent infectieux causant la pasteurellose (qui pourrait quand même être présent dans l'environnement de l'animal) peut devenir résistant au médicament. Il faut envisager ensuite les risques de toxicité. Tout médicament présente des risques d'effets secondaires qui peuvent se révéler franchement toxiques dans certaines situations. Par ailleurs, la santé publique est à considérer. Idéalement, certaines maladies ou même certains traitements devraient conduire à un retrait total de produits (lait, viande, etc.) du commerce. Cependant, étant donné les manquements des systèmes de vérification, les éleveurs (conscients de leurs pertes éventuelles) peuvent frauduleusement mettre ces produits sur le marché. Enfin, il faut évoquer les coûts: pertes matérielles dues à la diminution de la production, production de nouvelles thérapies, etc. Ces questions matérielles se posent notamment pour l'industrie pharmaceutique qui doit investir dans la recherche de nouveaux traitements pour contrer la perte d'efficacité des médicaments utilisés contre les différents agents infectieux.

Passons à présent à la troisième question portant sur la perception, dans les deux groupes envisagés, de la gravité des maladies réputées répandues dans la région dans laquelle l'étude a été menée - perception mise en évidence par le classement des noms de maladies (tableau 3). À cet égard, les résultats se sont montrés plutôt rassurants. Du côté des éleveurs, les cinq premières maladies (maladies que les éleveurs catégorisent dans ce qu'ils appellent "la première classe») sont les mêmes que celles de la liste des vétérinaires et, à une exception près, classées dans le même ordre. Les vétérinaires sont d'avis que, mis à part ces cinq maladies, l'ordre de gravité n'a pas d'importance parce que ces maladies ne sont pas dangereuses. Donc, de part et d'autre, la perception de la gravité des maladies les plus répandues est très similaire.

Un cas particulier est représenté par la maladie nommée Bodewu (peste bovine). On notera que même si les vétérinaires la mentionnent, elle est, pour eux, déjà éradiquée, contrairement aux éleveurs qui ont affirmé que cette maladie existe toujours, et c'est pour cela qu'elle apparaît en première position dans les tableaux 1 et 3 . Les problèmes évoqués plus haut (résistance, toxicité, ressources matérielles, etc.) peuvent ainsi se poser si des traitements inadéquats sont employés en raison d'un diagnostic fautif. Le fait qu'il existe des points de vue différents sur le statut d'une maladie rappelle nos propos antérieurs sur le rôle possible des termes, ainsi que d'autres 
facteurs, dans l'évaluation des interventions (Antia et Yassin 2001): comment la maladie dite «éradiquée» a-t-elle été désignée par les responsables de l'évaluation lors de l'enquête sur le terrain? Des pressions ont-elles été exercées pour dresser un bilan positif?

\section{Conclusion}

Nous estimons avoir démontré le potentiel de dysfonction communicationnelle attribuable aux questions terminologiques dans le contexte de l'élevage, secteur qui mérite bien d'être qualifié de stratégique et même de critique en matière de sécurité, en raison d'enjeux considérables, qui vont de la santé publique à un ensemble d'objectifs sociaux, y compris la sécurité alimentaire et la sécurité des emplois. Qui dit terminologique, dit non seulement conceptuel, mais aussi contexte social.

L'objectif modeste que se fixent les projets en communication pour le développement, c'est de sensibiliser les décideurs, les réalisateurs de projets et les personnes en général au fait que, parfois, les changements jugés unanimement nécessaires ne requièrent pas des investissements chiffrés en milliards de dollars. Par le biais des analyses terminologiques, nous croyons avoir montré que parler un même langage est déjà un pas important vers un approvisionnement sécuritaire en protéines et la protection de la santé du public.

Dans une publication antérieure (Antia et Yassin 2001), nous signalions, au terme de nos analyses, dix projets pour lesquels on pouvait apporter un soutien aux terminologues et à d'autres langagiers intéressés par la santé publique. Soulignons l'importance de l'analyse de la valeur prédictive des termes, à laquelle se joint naturellement le caractère essentiel de la spécificité de ces derniers à l'égard des concepts, autrement dit de la biunivocité.

\section{REMERCIEMENTS}

La rédaction de cet article a été facilitée par une bourse d'excellence de l'Agence universitaire de la Francophonie octroyée à Bassey E. Antia pour mener des recherches à l'Université de Montréal, Département de linguistique et de traduction, Groupe de recherche en sémantique, lexicologie et terminologie (GRESLET). Que soient vivement remerciés l'Agence universitaire de la Francophonie et le Pr André Clas (directeur du GRESLET au moment où ce travail a été réalisé). Pour leurs suggestions et commentaires, nous tenons à remercier aussi la Pre Sylvie Vandaele, Anaïs Tatossian, les relecteurs de la revue Meta ainsi que le Dr Chioma Ofoegbu.

\section{RÉFÉRENCES}

Antia, Bassey Edem et Kwasari, Andrew (2009, sous presse): Stories of chickens and dogs: a narrative metaphor for the analysis of encounters in the veterinary clinic. Communication and Medicine 6(1).

Antia, Bassey Edem (2000): Terminology and Language Planning: An Alternative Framework of Discourse and Practice. Amsterdam: John Benjamins.

Antia, Bassey Edem et Yassin, Khaled (2001): Coming to terms with the patient's terms on the patient's terms: terminology in public health. In: Felix MAYER, dir. Language for Special Purposes: Perspectives for the New Millennium. Tome 2. Tübingen: Gunter Narr, 871-881.

Arntz, Reiner et Picht, Heribert (1995): Einführung in die Terminologiearbeit. Hildesheim: Georg Olms. 
BULLETIN DES AGRICULTEURS (2002): Rapport accablant sur la gestion de l'épizootie de fièvre aphteuse en 2001. Consulté le $1^{\text {er }}$ juin 2009, <http://www.lebulletin.com/informations/ actualite/article.jsp?content=20020128_actualite_020128zg $>$.

Fraser, Colin et Restrepo-Estrada, Sonia (1998): Communicating for Development: Human Change for Survival. London: I.B. Tauris.

SÉCURITE PUBLIQUE CANADA (2006): Crise de fièvre aphteuse au Royaume-Uni - Répercussions sur l'infrastructure essentielle. Consulté le $1^{\text {er }}$ juin 2009, $<$ http://www.securitepublique.gc.ca/prg/em/fmd-fr.asp $>$. 